\title{
THE DISSOCIATION POINTS OF SOME CHLORIDES
}

\author{
BY PETER FIREMAN AND E. G. POR'TNER
}

When potassium or ammonium iodide is brought closely to the fumes given off by antimony pentachloride, a reddish-brown precipitate forms on the surface of the iodide. If the pulverized iodide is spread over a strip of white paper or a piece of porcelain the reaction presents the beautiful phenomenon of a cloud passing rapidly over the surface prepared for it and leaving a charred appearance in its track.

We had frequent occasion to observe this phenomenon in a study of the action of the iodides mentioned on antimony pentachloride, and the idea gradually impressed itself upon us that we were confronted with a rather interesting chemical reaction, that the reddish-brown superficial layer - obviously free iodine - might be separated by free chlorine, due to the dissociation of antimony pentachloride at ordinary temperature. It appeared of interest to look more closely into this matter.

First of all we confirmed, by the potassium iodide and starch test, that the reddish-brown precipitate was really free iodine. A like precipitate, spreading also like a cloud, is formed when free chlorine is allowed to pass over powdered potassium iodide. In both cases the thin reddish-brown layer volatilizes in a short time.

On the other hand, we found that when powdered potassium iodide is dropped into antimonic chloride the reaction, whatever it may be, proceeds at ordinary temperature only to a very slight extent, and that if the bodies are heated in a sealed tube at not too high temperatures $\left(120^{\circ}-140^{\circ}\right)$ antimony pentiodide is copiously formed. ${ }^{x}$

Now antimony pentiodide which has scarcely been prepared in a state approaching purity, is known to be a most unstable body, rapidly decomposing with the liberation of iodine. It

\footnotetext{
${ }^{1}$ In a future communication this subject will be fully treated.
} 
might be supposed that such is the origin of the reddish-brown precipitate on potassium iodide in the presence of fumes of antimonic chloride.

Again, the iodine could be conceived as resulting through the action of moisture, the latter starting a series of reactions (formation of hydrochloric acid, setting free of hydriodic acid, and breaking up of the latter) terminating in the separation of iodine.

In either of these cases, however, the instantaneous spreading of the reddish-brown precipitate on potassium iodide, with "the cloud effect" would remain unaccounted for. Moreover, if the iodine were the product of the decomposition of antimonic iodide, appreciable traces of antimonious iodide should be left on the volatilization of the iodine, which is not the case.

The most plausible conclusion from the above observations seems to be that the iodine is liberated by free chlorine, due to antimony pentachloride, or, at least, its vapor, being dissociated at ordinary temperatures. If this conclusion is correct, then such compounds as stannic chloride or titanium tetrachloride, which fume in the air like antimony pentachloride, but which are not known to dissociate, should not give the "cloud effect" on potassium iodide (free iodine), while such as phosphorous pentachloride should behave like antimonic chloride.

To begin with stannic chloride. The fumes of the latter acting on powdered potassium iodide turn it first yellow, then orange-yellow, and lastly, orange-red. Obviously, some stannic iodide is formed. On moist potassium iodide starch paper the fumes produce no effect on short exposure; only after a few minutes a slight blue coloration, which may be considered as resulting from the decomposition of hydriodic acid, formed either by the action of hydrochloric acid on potassium iodide, or by that of moisture on stannic iodide, or by both.

Titanium tetrachloride acts less readily on potassium iodide, with or without starch solution. . Even copious fumes of the chloride do not seem to affect either of the reagents named. Only when some drops of the titanium compound are poured 
over pulverized potassium iodide, some titanium tetriodide is formed, which first appears as a light brown precipitate turning soon darker, then reddish-brown; after some time the potassium iodide is left with a yellow tinge. On moist potassium iodide starch paper liquid titanium tetrachloride gives a blue coloration, along with a light-brown precipitate.

On the other hand, phosphorus pentachloride acts on dry potassium iodide as well as on a solution of the latter and starch, exactly as antimonic chloride does, provided it is heated. In other words, if antimony pentachloride is dissociated at ordinary temperatures, phosphorus pentachlorice is so only at higher temperatures. The experiments are suitably carried out in a test-tube and either of the reagents is held over its month.

Like the pentachlorides of antimony and phosphorus, behave also ferric, cupric, and chromic chloride. In all these cases heat has to be applied, but by far not such high temperatures are required as one would be inclined to suppose. Any way, the fact of the dissociation of the chlorides just mentioned can most readily be demonstrated by a very simple test-tube experiment.

It occurred to us of interest, and as feasible, to ascertain the lowest temperatures at which the chlorides under consideration show distinctly the fact of their dissociation; to ascertain - let us use the term - their "dissociation points."

We attempted to determine the dissociation points by bringing the substances into test-tubes in sufficient quantity to bury the bulb of the thermometer in them, and then heating in a bath of paraffin or fusible metal. In these experiments, however, moist potassium iodide starch paper was used to detect the first traces of chlorine.

Phosphorus pentachloride sublimes at about $100^{\circ}$, condensing a short distance above the level of the bath, and shows the first signs of chlorine at $157^{\circ}-15^{\circ}$. The decomposition seems to set in energetically, judging from the rapidity with which the entire surface of the test paper becomes blue. After a few minutes, no more free chlorine can be detected at the mouth of the test-tube, the temperature, however, remaining constantly at 
abont $158^{\circ}$ for a long time. On forcing the temperature higher, chlorine again escapes from the tube. Apparently, phosphorus trichloride, one of the products of the dissociation, on distilling and condensing on the ring of sublimed phosphorus pentachloride, absorbs, after a while, the chlorine passing upward.

The same results were obtained with phosphorus pentachloride from different lots, and in short and long test-tubes alike.

Ferric chloride, anhydrous, begins to dissociate at $122^{\circ}-$ I $23^{\circ}$. To obtain a constant dissociation point the ferric chloride must be well dried, otherwise the decomposition begins at considerably higher temperatures. Also, in this case, the experiments were carried out in both short and long test-tubes with the same result. Victor Meyer $^{2}$ and Grünbaum could discover ferrous chloride as a product of the decomposition of ferric chloride only after the latter had been heated above $44^{\circ}$.

Cupric chloride, anhydrous, shows the first signs of dissociation at $344^{\circ}$.

Chromic chloride, anhydrous, begins to dissociate at $355^{\circ}$.

We think that by the cumulative evidence in the foregoing, we have established beyond a doubt that in the experiments described above with the higher chlorides of antimony, phosphorus, iron, copper, and chromium, we were confronted with the same reaction, that of dissociation.

In further support of this view, we want to emphasize the following points :

In the cases of cupric and chromic chloride, everybody must at once admit that the reaction in question cannot be anything else but a pure dissociation, it being quite impossible for these chlorides, as well as the lower ones to which they are teduced, to reach the mouth of the test-tube at the temperature indicated above.

And the apparent reaction is in every way the same, also, in the cases of the chlorides of antimony, phosphorus, and iron;

${ }^{1}$ Ber. chem. Ges. Berlin, 21, 687 (1888). 
this uniformity is remarkable and suggestive of a common nature.

Lastly, the constant temperature at which the chlorine reaction sets in, in each case, is weighty evidence in favor of the view propounder. It need not be overlooked in this connection that the constancy of what we call "the dissociation point" is the more significant in view of the fact that it is independent of the length of the test-tube in which the reaction is carried out; this constancy of temperature would be out of the question if a volatilization of the chlorides and their coming in contact with the potassium iodide were necessary to initiate the reaction.

We believe to have indicated a simple general test for the dissociation of chlorides; a test well suited for demonstration in the lecture. We further believe to have determined the "dissociation points" of a number of chlorides, being the first data for establishing a new constant.

We intend to pursue the subject further.

Alexandria, $V a$. 